\title{
Users' Perceptions of an in-Home Electronic Medication Dispensing System: A Qualitative Study
}

This article was published in the following Dove Press journal:

Medical Devices: Evidence and Research

\author{
Armghan Ahmad \\ Venus Chiu \\ Mubashir Aslam Arain \\ Alberta Health Services, Health Systems \\ Evaluation and Evidence, Calgary, $A B$, \\ Canada
}

Background: Managing and taking multiple medications as prescribed can be a difficult task for older adults. In-home medication dispensing technologies could help enhance care. The objective of the study was to determine users' perspectives on a medication dispensing system (MDS) in supporting medication adherence of individuals living at home with chronic conditions.

Methods: This analysis is a part of a randomized controlled trial on an MDS in a Western Canadian province. We interviewed participants who were recruited into the intervention group and started using an MDS. A maximum variation purposive sampling was used to select interview participants based on age, number of medications, and health conditions.

Results: Thirteen participants were interviewed; most participants were females $(n=11)$ and the average age was $63.7(\mathrm{SD}=8.2)$ years with an average of $8.9(\mathrm{SD}=3.6)$ prescribed medications. The most common health conditions were hypertension, diabetes, arthritis, and anxiety and depression. Four main themes emerged from thematic analysis: MDS acceptability, MDS patient support, need for the MDS, and areas of technology improvement. Most of the participants found the MDS to be acceptable and convenient, although privacy and security was an issue for some older adults. Audio and visual reminders and preorganized medication supported participants' medication adherence and independence in daily routines. The perceived necessity of the MDS was split among participants with cost being one of the main concerns. Areas of technology improvement included the hard-to-open plastic medication packets and the sometimes inexact recording of medication adherence by the MDS if medications were dispensed on behalf of the patients.

Conclusion: The MDS is an acceptable tool for improving medication management and adherence in older adults. Increased medication adherence may lead to patient and systemlevel benefits.

Keywords: medication dispenser, qualitative research methods, patient experience and preferences, aging and seniors, polypharmacy, chronic conditions

\section{Introduction}

Aging is connected with a higher prevalence of chronic morbidity, resulting in the need for complex medication regimens. Adherence to complex medication regimens is particularly challenging for older adults and seniors. Medication adherence refers to the extent to which patients take medications as prescribed by their healthcare providers. ${ }^{1}$ A review has reported around $26 \%$ to $59 \%$ of those 60 and above are noncompliant with their medication regimens. ${ }^{2}$ Multiple factors contribute to poor medication adherence, such as an increase in the number of medications and the frequency of medication intake, and a prolonged course of drug therapy. ${ }^{3}$ Nonadherence causes detrimental consequences; many chronic conditions can lead to
Correspondence: Mubashir Aslam Arain Alberta Health Services, Health Systems Evaluation and Evidence, I030I Southport Lane SW Calgary, AB T2W IS7, Canada

Tel + I 403-943-0783

$\mathrm{Fax}+$ I 403-943-2875

Email mubashiraslam.arain@ahs.ca 
complications, decrease patients' quality of life, and increase mortality. Given Canadian seniors age 65 and older are prescribed an average of 6.9 medications and one in four are prescribed 10 or more medications, ${ }^{4}$ nonadherence and medication management issues are significant challenges for optimizing patient outcomes. ${ }^{5,6}$

Multiple forms of behavioral and educational interventions exist with the aim to improve individuals' medication adherence and medication management. ${ }^{7}$ Technological interventions have also been increasingly adopted. These include electronic communication devices (e.g., phones, text messaging devices, pagers, and alarms $),{ }^{8}$ smartphone applications, ${ }^{9}$ and augmented reality systems. ${ }^{10}$ A recent technological development aimed to improve medication adherence is medication dispensing technology. These devices range in complexity, from systems that help organize medications and provide reminders to systems that additionally measure medication adherence and offer inperson intervention. ${ }^{11}$

The available research on medication dispensing technology use in older populations has largely focused on the technological impact on medication adherence and patient outcomes. The various functions of medication dispensing technologies have been shown to significantly improve medication adherence in community-dwelling older adults with chronic conditions. ${ }^{12-15}$ Studies suggest these kinds of technologies are superior to pill organizers and self-management of medication in helping older adults adhere to their medication regimens. ${ }^{12,15}$ Medication dispensing technologies have been shown to improve patient health outcomes, such as reduced frequency of physician visits and hospitalizations, and reduced prescribed medication overtime attributed to the stabilization of patients' conditions due to medication adherence. ${ }^{12,15}$ Although most evidence concurs that medication dispensing technologies enhance patient health outcomes, one study reported that the integration of medication dispensing technology to home-based nurse care coordination did not appear to improve frail older adults' health outcomes. ${ }^{16}$

A limited number of studies have examined the experience of community-dwelling older adults with other medication dispensing technologies. Older adults generally accept and are satisfied with using medication dispensing technologies, finding the technologies easy to use and helpful in reminding them about and managing their medication. ${ }^{14,17,18}$ Conversely, commonly perceived challenges of the technology include $\operatorname{cost}^{17,18}$ and lack of need or desire to use the technology due to physical abilities or disabilities. ${ }^{15,18}$ The views of older adults regarding medication dispensing technologies are divided with some patients finding fixed medication schedules incompatible with their lifestyle, ${ }^{14,18}$ while other patients believe the devices allow for flexibility and do not affect their daily activities. ${ }^{14,17}$

The home-based medication dispensing system (MDS) used in this study can monitor and assist older adults with managing their medications. The MDS is a technology that provides medication reminders, pill-dispensing, and other forms of integrated assistance, such as real-time pharmacy, caregiver, or healthcare support and intervention through telehealth capabilities. Understanding patients' perceptions and experiences with the MDS is essential to facilitate future development of the technology and improve medication adherence and health outcomes. The purpose of this study was to determine users' perspectives on the MDS to support medication adherence for individuals living at home with chronic conditions.

\section{Methods \\ Design}

This study was conducted from March 2019 to October 2019 in a Western Canadian province; it was part of a larger randomized controlled trial (RCT) on the MDS. A qualitative method was chosen to closely examine participants' views of the MDS. We interviewed participants recruited into the intervention group around a month after they started using the MDS. For the study period, the participants did not incur any cost for the use of the MDS. This research study was approved by the Research Ethics Board at the University of Alberta (Ethics ID: PRO 00087782).

\section{The Medication Dispensing System}

The intervention comprised of interactive tools to optimize medication adherence and clinical outcomes. Medication packets were pre-packaged by day and time of administration. The MDS dispensed medications on time with visual and audio reminders. The audio alert amplified if the plastic medication packet was not dispensed from the system. The MDS allowed participants to dispense their medications early if they were going to be away from home. The system also displayed personalized and condition-specific questions (clinical engagement questions) that provided pharmacists opportunities for remote patient intervention, if needed, through telecommunication. 


\section{Population and Sample}

The research team recruited study participants from a Family Care Clinic (specialized clinic for patients with complex care needs). The majority of the clinic clientele was of low socioeconomic status. Participants were recruited into the study based on predetermined eligibility criteria. The inclusion criteria included: 1) English speakers 2) age 50 and over 3) diagnosed with one or more chronic condition(s) and 4) currently taking five or more prescribed oral medications. Patients with moderate to severe cognitive impairment were excluded from the study as the MDS needs sufficient cognition and decisionmaking ability to operate safely. Healthcare providers in the Family Care Clinic prescreened potential study participants to determine if they were cognitively fit to provide research study consent and suitable to participate in the study prior to referring them to the on-site research team member for recruitment. We used maximum variation purposive sampling based on age, number of medications, and health conditions to select interview participants.

\section{Data Collection}

The research team conducted semi-structured interviews through telephone or in-person meetings with study participants. Participants were asked about their acceptance of technology, experience with and perception of using the MDS, and the impact the MDS had on their medication adherence and daily routines (Appendix S1). Participants provided written consent to be recorded and interviewed by the research team. Each interview lasted for 20-30 mins.

\section{Data Analysis}

Each recorded interview and corresponding transcription was assigned a unique ID. Thematic analysis was used to generate common themes while recognizing the context and uniqueness of the data. ${ }^{19}$ Inductive thematic coding of emerging themes was employed using NVivo 12..$^{20,21}$ Using this approach, two research team members conducted multiple close-readings of the transcripts, identified and coded recurring themes and patterns, and explored the possible relationships between themes and explanations. ${ }^{22}$ Research and interview questions were used to guide theme development. The interrater reliability between the two researchers was deemed excellent with a Kappa coefficient of $0.85 .{ }^{23}$ Consensus was further reached regarding any discrepancies in the identification of broader themes.

\section{Results}

\section{Demographic and Clinical Characteristics}

A total of 13 participants were interviewed; 12 were MDS users and one was a caregiver of a study participant. Participants interviewed were mostly females $(n=11)$ and the average age was $63.7(\mathrm{SD}=8.2)$ years. The majority of the participants identified themselves as Caucasian $(n=10)$. All participants were living independently in a community: half were living with a spouse $(\mathrm{n}=6)$, and another quarter were living alone in their own residence $(n=3)$. The most common chronic conditions among the participants were hypertension $(\mathrm{n}=8)$, diabetes $(\mathrm{n}=7)$, arthritis $(\mathrm{n}=7)$, and anxiety and depression $(n=6)$. All participants had two or more diagnosed chronic conditions and an average of 8.9 $(\mathrm{SD}=3.6)$ prescribed medications (Table 1).

Four main themes emerged from the thematic analysis of coded transcripts.

\section{MDS Patient Support}

\section{Support in Medication Management}

Participants perceived the alert function of the MDS as a reliable feature that kept them on track and on schedule for their medication regimen. Most participants found the reminders non-intrusive, helped them remember, and ensured they took their medications on time.

I was taking my medications on time, $99 \%$ of the time and it seems like my diabetic glucose [level] has gone under seven which is really, really good. So taking my pills on time is helping me with whatever the pills are supposed to do my body.

A few participants described the reminders as interruptive at times, especially when participants required or desired a certain level of flexibility in their schedule. Participants ignored or accidentally disregarded the reminders due to busyness. However, they understood the purpose behind the reminders and the impact on their medication commitment

Well, it reminds me because sometimes I have forgotten 'oh its lunch time, it's time to take medication' because I get busy sometimes. Sometimes, I have the tendency to say 'oh I'll go get my medication,' but on my way there I get involved in something else and then I forget so this kind of helps me .... you know, got to take it out and I take them usually right away.

The MDS assisted users by providing organized medications. It was considered more convenient and safer compared to other medication management methods (e.g., blister pack). 
Table I Demographic and Clinical Characteristics of Interviewed Participants ( $\mathrm{N}=12)$

\begin{tabular}{|l|l|}
\hline Characteristic & $\begin{array}{l}\text { Number of } \\
\text { Participants (\%) }\end{array}$ \\
\hline Gender & \\
Female & $11(91.7 \%)$ \\
Male & $1(8.3 \%)$ \\
\hline Age & $63.7(8.2)$ \\
$50-55$ years & $2(16.7 \%)$ \\
$56-60$ years & $3(25.0 \%)$ \\
$61-65$ years & $2(16.7 \%)$ \\
66-70 years & $3(25.0 \%)$ \\
$76-80$ years & $2(16.7 \%)$ \\
\hline Ethnicity & \\
Caucasian & $10(83.3 \%)$ \\
Aboriginal & $1(8.3 \%)$ \\
Southeast Asian & $1(8.3 \%)$ \\
\hline Living Arrangements & \\
With spouse & $6(50.0 \%)$ \\
Alone in own residence & $3(25.0 \%)$ \\
With family member(s) other than spouse & $2(16.7 \%)$ \\
With a roommate & $1(8.3 \%)$ \\
\hline Chronic Health Conditions & $8.9(3.6)$ \\
Hypertension & $8(66.7 \%)$ \\
Diabetes & $4(33.3 \%)$ \\
Arthritis & $7(66.7 \%)$ \\
Anxiety and depression & $7(58.3 \%)$ \\
Asthma & $6(50.0 \%)$ \\
Sleep apnea & $3(25.0 \%)$ \\
Chronic pain & $2(16.7 \%)$ \\
\hline Number of Prescribed Medications & $2(16.7 \%)$ \\
\hline
\end{tabular}

Note: *Mean and standard deviation reported for age and number of prescribed medications.

Participants with physical hindrance (e.g., arthritis) that challenged their ability to organize or take the medications themselves found it convenient and beneficial to their conditions.

It's just really convenient and I don't have to sort my meds. That's the nice thing, it's all done for me.

Even with the blister packs, I doubled up on [medication] during the heart failure ....You're just so sick and you're not there, right? With [the MDS] you can't do that, this is way safer, this is safe.

\section{Support in Daily Life and Routine}

Most participants reported that the MDS did not interfere with their life and, in fact, concreted their routines. Further, the feature that allowed participants to dispense their medication early provided them the ability to be mobile and feel more independent.

It probably helped me with the daily routine because I am more aware of it so if I'm going out, I stop and think 'am I going to miss [my medication]?' ... If I am, then I can get my meds to go.

The MDS resulted in perceived improvements in psychological and physical health. Participants shared that they felt less worried and stressed in their lives which helped improve relationships with their family members. In one case, both the caregiver and the care receiver perceived reduced caregiver burden because the participant was taking medications by herself and on time. Another participant specifically attributed her health improvement to the help of the MDS.

I really didn't think I needed [the MDS] and so I really didn't have a lot of expectations that [it] was going to make such a difference but I really have noticed that 'yes I am taking my pills on time, yes I am remembering to take them'. I am feeling better because of taking my pills at the proper intervals.

So yeah it worked well for us, I'm not as upset, it's [a] better relationship between [my husband] and I, it's just something's been less stressful my part or his part.

\section{MDS Acceptability Usability of the MDS}

Overall, participants were either highly receptive or neutral to using the MDS to manage their medications. Participants liked the usability of the MDS and found it easy to learn and use.

It's really easy to use and I'm not a really techy person but I found [the MDS] quite easy to use which is good.

The caregiver also positively perceived the usability of the device.

It's easy technology for her [the care reciver] to understand like before if I just show her anything, say on a tablet or a computer, like she wouldn't really get [it] whatsoever but I showed her maybe once, twice on this and now she just does it by herself.

\section{Privacy and Security}

One area that perturbed participants' positive attitudes towards using the MDS was privacy and security issues. Medication refill boxes were delivered to participants' 
homes on a weekly or bi-weekly basis by the pharmacy. Each medication packet was labeled with the participant's name, date and time of administration, medication names, and dosage. A couple of participants voiced their concerns regarding the personal and medication information disclosed on the medication packets, refill boxes, and prompted clinical engagement questions. One participant specifically believed that the presence of and the display on the device were a form of privacy disturbance.

It's shoved in my bedroom because I think, psychologically, it's not good. It looks like you are sick, I don't want people to see.

\section{Need for the MDS}

About half of the participants perceived the MDS to be useful but not necessary. Participants who did not perceive a need for the MDS believed it was because their mental and physical faculties were well enough for managing their own medication. However, the same participants believed they would probably need the MDS in the future. They recommended the technology to anyone, old or young, with problems organizing and taking medications and difficulty remembering to take medications due to aging or sickness. In parallel, participants who perceived a need for the MDS ascribed it to the state of their declining health or their inability to manage their medications due to the daily impact of their chronic health conditions.

I do have a need for [the MDS] because my hands are not getting better, it's getting worse.

When participants were asked to rate (on a scale of 1-10) their likelihood to recommend the MDS to another older adult to help with medication management, all participants strongly recommended the device, with 10 participants giving a score of 10/10 and the rest giving 9/10. When asked about their satisfaction with the device since the initial use, the ratings were similarly favorable, with 10 participants being very satisfied (10/10). The lowest reported score was $8 / 10$ by two participants. The main reasons behind any deviation from a full rating for both questions were the lack of necessity participants perceived for themselves and, potentially, for others and the cost of leasing the device after the study period. The $\$ 100 \mathrm{CAD}$ monthly leasing fee after the study period was a barrier for continuing to use the technology for most participants.
I think [the MDS] is worthwhile, and I think it's a great thing for people to have but the cost of it [is] way too high for a lot of people.

\section{Areas of Improvement Medication Packets}

Most of the participants mentioned that the plastic medication packets dispensed by the MDS were not easy to open using their fingers as intended. Opening the medication packets was particularly challenging for participants with physical hindrances. Thus, many participants defaulted to using scissors or their teeth to make a tear.

Well, sometimes I find it really hard to open the packets.... And sometimes my hands aren't quite strong enough to make the original tear in them so I generally end up going and find a pair of scissors.

The only thing is the opening the packets sometimes, they don't pull open when you need it. I got some arthritis in my hands so some days it's hard.

\section{Measure of Medication Adherence}

As the MDS recorded medication adherence based on the assumption that users took their medications immediately after they dispensed the packets, some participants suggested that the MDS may not always be accurate in tracking adherence. MDS users may choose to administer the medications at a later time than it was dispensed, especially if a family member or caregiver dispensed medications on their behalf.

If [the MDS] thinks that I'm taking my pills at six o'clock, some days [it]'s dead wrong because all we've done is taken them out of the machine.

\section{Discussion}

Following the perceptions of the participants, it can be concluded that the MDS was useful for supporting the management of and adherence to medications. Supports provided through pre-organized medications and reminders were convenient for participants and reduced psychological distress. Blister pack packaging is a widely used medication management method that pre-organizes medications and aims to help patients maintain their prescribed dosing schedules. ${ }^{24}$ However, the blister pack packaging method alone does not support improved medication adherence. A systematic review concluded that blister pack packaging when combined with education and other 
reminder strategies may improve medication adherence. ${ }^{24}$ The MDS used in this study provided that functionality, and perceived usefulness by most participants was mainly due to pre-organized medications and reminders.

The MDS helped to maintain and improve individuals' daily routines and independence. The MDS had the functionality that allowed participants to dispense medications early. This contrasted with a previous study that reported mobility as an issue for some users. ${ }^{18}$ However, this is explained by the fact that the medication dispensing technology used in the previous study did not provide the same early-dispensing flexibility as the MDS. With the current trend towards "aging in place" - to remain living independently in individuals' own community ${ }^{25}$ - the portability of medications from the MDS and other medication management methods would be crucial for older adults to maintain their autonomy over time.

The perceptions of the participants demonstrated that the MDS was generally accepted by older adults taking multiple medications for their chronic conditions. Older adults tend to be late adopters of technology compared to their younger counterparts. ${ }^{26}$ Thus, the simple and easy process of using an MDS was crucial for obtaining the high technology acceptance among users. Literature suggests that any anticipated concerns with security and privacy could be barriers to individual (and societal) acceptance and adoption of assistive technologies. ${ }^{27-29}$ This is reflected by some participants' reports concerning the appearance of personal information on medication refill boxes and medication packets dispensed through the MDS. The demand for privacy and security by interview participants was somewhat expected as one study found females and healthy adults demanded higher standards compared to males and frail adults. ${ }^{27}$ The associated pharmacies or technology companies may need to further cater product designs and placement of personal and medication-related information to fit both the security desire of patients and medication packaging standards. On the other hand, the physical presence of the MDS in common areas of homes may be perceived as a form of privacy violation because it creates an image of sickness for users and for others that see the device in users' homes. This challenge could be easily resolved by relocating the device to a private space.

All participants highly recommended the system for others but believed it would be more useful for individuals with chronic conditions that physically or cognitively challenged them to adhere to their medications. Individuals with cognitive impairment are at a higher risk for medication non-adherence as cognitive ability and processes are needed for medication management. ${ }^{30}$ A systematic review reported medication adherence among cognitively impaired individuals ranges from $10.7 \%$ to $38.0 \%{ }^{30}$ The study demonstrated memory assistive devices (such as medication dispensing technologies) are used more often by cognitively impaired individuals and result in increased adherence. ${ }^{30}$ Also, evironmental cues associated with the repetitive task of taking medication were reported to have contributed to increased adherence. ${ }^{30}$ Therefore, the MDS or related technologies may be even more beneficial for these high-risk patients where audio and visual reminders provide repetitive environmental cues. Further, the MDS and related technologies should be targeted to consumers residing at supportive living facilities as these patients tend to have higher needs and care requirements.

The post-study monthly out-of-pocket cost was perceived as a barrier for almost all participants, even for individuals that strongly believed they had a need for the technology. A study conducted on another medication dispensing technology also found cost as a barrier for future use and indicated one participant was in-favor of using the device if costs were covered. ${ }^{17}$ Patients would have to evaluate if the extra cost is a better alternative to sorting medications, remembering to take medications, and experiencing negative consequences related to medication non-adherence.

The findings from this study add value to the existing literature regarding future use and adoption of the MDS and related medication dispensing technologies. It is evident that the treatment of chronic conditions not only requires coordinated team-based care involving various healthcare providers but also includes the long-term use of pharmacotherapy. ${ }^{3}$ It is known that $50 \%$ of chronic condition patients are non-adherent to their medication regimens, despite the overwhelming awareness of the benefits associated with medication adherence. ${ }^{31}$ As the study findings and literature suggest, many participants miss their medication times unintentionally due to factors related to forgetfulness or busyness. ${ }^{24}$ Based on the participants' perceptions and experiences, the MDS seems to be a valuable support system for users to optimize pharmacotherapy commitments and health benefits. This has significant implications for the healthcare system as nearly $10 \%$ of hospitalizations in older adults are caused by medication non-adherence. ${ }^{32}$ The MDS or other related technologies should be utilized by the healthcare system 
to reduce medication-related non-adherence costs while promoting trends toward aging in place.

\section{Product Design Improvements}

A couple of areas of product design improvement arose in the interviews. The user-related challenge was that the dispensed medication plastic packets were not easy to open for most of the participants. Participants were mostly using scissors or their teeth to open the packets. A study investigated the relationship between blister pack design and utilization problems among older adults and determined that opening force and opening mechanism can have a significant impact on the usability of blister packs. ${ }^{33}$ The manufacturers of the MDS should consider incorporating a small cutting blade that will cut the packets on the edge before dispensing medications.

A few of the participants living with a spouse or a family member reported that their medications were dispensed by someone else to silence the alerts. The MDS assumed 100\% adherence as soon as medications were dispensed. The manufacturers of the MDS can potentially enhance the safety and measurement of the system by only dispensing medications based on the finger-print scan of the registered user. In order to silence the system, there should be an option on the touch screen, such as "user not home", where reminders would be delayed for one or $2 \mathrm{hrs}$ based on the second prompted question "when will the registered user be home?".

\section{Limitation}

First, the participants in the study were interviewed around a month after they started using the MDS. As the participants were in the early stages of technology adoption and usage, they might not have utilized the full features of the MDS. Moreover, the currently perceived benefits of the MDS could be a result of the Halo effect or participants' initial positive impression towards the product. Second, the participants were recruited from only one Family Care Clinic and the majority of its clientele was of low socioeconomic status. The perceptions related to system cost may vary based on socioeconomic status and financial situation. Third, the majority of the participants interviewed were Caucasian females. The literature suggests, in general, females are prescribed more medications than males and there is a significant difference between gender regarding medication use and adherence. ${ }^{34}$ Likewise, gender variations in perceptions of the MDS may have influenced the results of the study regarding the acceptability of the MDS, in particular concerns related to privacy and security. Genderbased differences in medication use may contribute to poorer medication adherence for females, resulting in perceived higher need and benefits related to the MDS. Therefore, the current sample may not be representative of the population and individuals need to be cautious in generalizing the results.

\section{Future Research Direction}

The research team will be conducting interviews with the same participants 6 months after they started using the MDS to understand if the current positive experience is sustained and any new benefits and challenges of the technology. The RCT will provide quantitative evidence regarding the MDS's actual (rather than perceived) effectiveness in influencing patient medication adherence and health outcomes. Future research should examine diverse demographics to understand the different perceptions of the MDS, or lack thereof, and increase the generalizability of the results of the current study.

\section{Conclusion}

The MDS is an acceptable tool for improving medication adherence in older adults. The audio and visual alerts remind users to take medications on time and maintain consistent medication schedules. The technology supports complex care need patients to improve medication adherence while living independently in the community. With a few improvements to the current technology, the MDS has the potential to bring patient and system-level benefits.

\section{Ethics Approval and Consent to Participate}

This research study was approved by the Research Ethics Board at the University of Alberta (Ethics ID: PRO 00087782). Written consent was obtained from each participant. Moreover, all data collection, management, and storing procedures complied with the Health Information Act and the Freedom of Information and Privacy Act. All participants were provided with information on the research study and how the data would be used.

\section{Availability of Data and Material}

The interview transcripts coded and analyzed during the current study are not publicly available due to ethics requirements and organizational data sharing restrictions. 


\section{Acknowledgments}

We would like to thank the staff at the East Calgary Family Care Clinic for their support during the recruitment period. Special thanks to Carrie Sauve (Patient Care Manager) and Olivia McCreary (Analyst) for promoting the study at the clinic and having eligible patients informed by the providers on-site or through the mailed invitation letters. We also appreciate the help from Catalyst Healthcare Ltd. and Sandstone Pharmacies Ltd. staff for mobilizing the study through on-boarding of patients for the MDS, delivery of medications, and ongoing technical support. The authors would like to acknowledge all the professional members mentioned below as collaborators; these individuals were actively engaged and played a key role in the study milestones and successes.

Dr. Brian M. Cornelson, Medical Director, East Calgary Family Care Clinic, Alberta Health Services

Kasumi Oda, Vice President of Marketing, Catalyst Healthcare Ltd.

Kristen Antunes, Director of Clinical Outcomes, Catalyst Healthcare Ltd.

Julie McRae, Former Director of Clinical Outcomes, Catalyst Healthcare Ltd.

Bruce Winston, President, Sandstone Pharmacies (A division of APEX Pharmacies Ltd.)

Jill Mason, Director of Clinical Services, Sandstone Pharmacies (A division of APEX Pharmacies Ltd.)

\section{Author Contributions}

All authors contributed to data analysis, drafting or revising the article, gave final approval of the version to be published, and agree to be accountable for all aspects of the work.

\section{Funding}

The study was funded by the Centre for Aging and Brain Health Innovation (CABHI) through its Industry Innovation Partnership Program $\left(\mathrm{I}^{2} \mathrm{P}^{2}\right)$. CABHI is a governmentfunded program focused on improving seniors' health through innovation themes of Aging in Place, Caregiver Support, Care Coordination and Navigation, and Cognitive Health. Catalyst Healthcare Ltd. was awarded the grant as the MDS addressed CABHI innovation themes and priority areas.

\section{Disclosure}

$\mathrm{AA}, \mathrm{VC}$, and MAA report grants from the Centre for Aging and Brain Health Innovation (CABHI), during the conduct of the study. The authors report no other conflicts of interest in this work.

\section{References}

1. Osterberg L, Blaschke T. Adherence to medication. $N$ Eng $J$ Med. 2005;353(5):487-497. doi:10.1056/NEJMra050100

2. van Eijken M, Tsang S, Wensing M, de Smet PA, Grol RP. Interventions to improve medication compliance in older patients living in the community. Drugs Aging. 2003;20(3):229-240. doi:10.2165/00002 512-200320030-00006

3. Brown MT, Bussell JK. Medication adherence: WHO cares? Mayo Clinic Proc. 2011;86(4):304-314. doi:10.4065/mcp.2010.0575

4. Canadian Institute for Health Information. Drug use among seniors in Canada 2016. Ottawa, ON: CIHI; 2018.

5. Stirratt MJ, Curtis JR, Danila MI, Hansen R, Miller MJ, Gakumo CA. Advancing the science and practice of medication adherence. $J$ Gen Intern Med. 2018;33(2):216-222. doi:10.1007/s11606-017-4198-4

6. Sheehan OC, Kharrazi H, Carl KJ, et al. Helping older adults improve their medication experience (home) by addressing medication regimen complexity in home healthcare. Home Healthcare Now. 2018;36(1):10-19. doi:10.1097/NHH.0000000000000632

7. George J, Elliott RA, Stewart DC. A systematic review of interventions to improve medication taking in elderly patients prescribed multiple medications. Drugs Aging. 2008;25(4):307-324. doi:10.2165/00002 512-200825040-00004

8. Granger BB, Bosworth H. Medication adherence: emerging use of technology. Curr Opin Cardiol. 2011;26(4):279. doi:10.1097/HCO. $0 \mathrm{~b} 013 \mathrm{e} 328347 \mathrm{c} 150$

9. Dayer L, Heldenbrand S, Anderson P, Gubbins PO, Martin BC. Smartphone medication adherence apps: potential benefits to patients and providers. J Am Pharm Assoc. 2013;53(2):172-181. doi:10.1331/ JAPhA.2013.12202

10. Guerrero E, Lu MH, Yueh HP, Lindgren H. Designing and evaluating an intelligent augmented reality system for assisting older adults' medication management. Cogn Syst Res. 2019;58:278-291. doi:10.1016/j.cogsys.2019.07.001

11. Paterson M, Kinnear M, Bond C, McKinstry B. A systematic review of electronic multi-compartment medication devices with reminder systems for improving adherence to self-administered medications. Int J Pharm Pract. 2017;25(3):185-194. doi:10.1111/ijpp.12242

12. Winland-Brown JE, Valiant J. Effectiveness of different medication management approaches on elders' medication adherence. Outcomes Manag Nurs Pract. 2000;4(4):172-176.

13. Kamimura T. Older adults with Alzheimer's disease who have used an automatic medication dispenser for 3 or more years. Clin Gerontol. 2019;42(1):127-133. doi:10.1080/07317115.2017.1347594

14. Suzuki R, Hasegawa T. Evaluation of a one-dose package medication support system for community-based elderly patients and eldercare facilities. Bulletin Am Soc Hosp Pharm. 2018;75(9):e202-e212.

15. Buckwalter KC, Wakefield BJ, Hanna B, Lehmann J. New technology for medication adherence: electronically managed medication dispensing system. J Gerontol Nurs. 2004;30(7):5-8. doi:10.3928/ 0098-9134-20040701-04

16. Marek KD, Stetzer F, Ryan PA, et al. Nurse care coordination and technology effects on health status of frail elderly via enhanced self-management of medication: randomized clinical trial to test efficacy. Nurs Res. 2013;62 (4):269. doi:10.1097/NNR.0b013e318298aa55

17. Reeder B, Demiris G, Marek KD. Older adults' satisfaction with a medication dispensing device in home care. Inf Health Social Care. 2013;38(3):211-222. doi:10.3109/17538157.2012.741084

18. Allemann SS, Hersberger KE, Arnet I. Patient views on an electronic dispensing device for prepackaged polypharmacy: A qualitative assessment in an ambulatory setting. Integr Pharm Res Pract. 2015;4:167. doi:10.2147/IPRP.S90923 
19. Braun V, Clarke V. Using thematic analysis in psychology. Qual Res Psychol. 2006;3(2):77-101. doi:10.1191/1478088706qp063oa

20. Thomas DA. General inductive approach for analyzing qualitative evaluation data. Am J Eval. 2006;27(2):237-246. doi:10.1177/ 1098214005283748

21. NVivo qualitative data analysis software. QSR International Pty Ltd. Version 12. 2018.

22. Morse J, Richards L. Read Me First for a User's Guide to Qualitative Methods. Sage Publications; 2002.

23. Landis JR, Koch GG. The measurement of observer agreement for categorical data. Biometrics. 1977;1:159-174. doi:10.2307/2529310

24. Zedler BK, Kakad P, Colilla S, Murrelle L, Shah NR. Does packaging with a calendar feature improve adherence to self-administered medication for long-term use? A systematic review. Clin Ther. 2011;33 (1):62-73. doi:10.1016/j.clinthera.2011.02.003

25. Davey JA, de Joux V, Nana G, Arcus M. Accommodation Options for Older People in Aotearoa/New Zealand. Christchurch: Centre for Housing Research; 2004.

26. Fischer SH, David D, Crotty BH, Dierks M, Safran C. Acceptance and use of health information technology by community-dwelling elders. Int $J$ Med Inform. 2014;83(9):624-635. doi:10.1016/j.ijmedinf.2014. 06.005

27. Thierer AD. The internet of things and wearable technology: addressing privacy and security concerns without derailing innovation. Rich JL Tech. 2014;21:1.
28. Wilkowska W, Ziefle M. Privacy and data security in E-health: requirements from the user's perspective. Health Informatics J. 2012;18(3):191-201. doi:10.1177/1460458212442933

29. Young R, Willis E, Cameron G, Geana M. "Willing but unwilling": attitudinal barriers to adoption of home-based health information technology among older adults. Health Informatics J. 2014;20 (2):127-135. doi:10.1177/1460458213486906

30. Smith D, Lovell J, Weller C, et al. A systematic review of medication non-adherence in persons with dementia or cognitive impairment. PLoS One. 2017;12(2):e0170651. doi:10.1371/journal.pone.0170651

31. Tamblyn R, Eguale T, Huang A, Winslade N, Doran P. The incidence and determinants of primary nonadherence with prescribed medication in primary care: A cohort study. Ann Intern Med. 2014;160 (7):441-450. doi:10.7326/M13-1705

32. Iuga AO, McGuire MJ. Adherence and health care costs. Risk Manag Healthc Policy. 2014;7:35-44. doi:10.2147/RMHP.S19801

33. Mühlfeld L, Langguth $P$, Häusler H, Hagels H. Influence of blister package design on usability among older adults. Int J Clin Pharm. 2012;34(4):553-560. doi:10.1007/s11096-012-9643-1

34. Manteuffel M, Williams S, Chen W, Verbrugge RR, Pittman DG, Steinkellner A. Influence of patient sex and gender on medication use, adherence, and prescribing alignment with guidelines. $J$ Women's Health. 2014;23(2):112-119. doi:10.1089/jwh.2012.3972
Medical Devices: Evidence and Research

\section{Publish your work in this journal}

Medical Devices: Evidence and Research is an international, peerreviewed, open access journal that focuses on the evidence, technology, research, and expert opinion supporting the use and application of medical devices in the diagnosis, monitoring, treatment and management of clinical conditions and physiological processes. The identification of novel devices and optimal use of existing devices which will lead to improved clinical outcomes and more effective patient management and safety is a key feature of the journal. The manuscript management system is completely online and includes a very quick and fair peer-review system. Visit http:// www.dovepress.com/testimonials.php to read real quotes from published authors. 